\title{
Outcomes based on plasma biomarkers in METEOR, a randomized phase 3 trial of cabozantinib vs everolimus in advanced renal cell carcinoma
}

Thomas Powles ${ }^{1 *}$, Toni K. Choueiri ${ }^{2}$, Robert J. Motzer ${ }^{3}$, Eric Jonasch ${ }^{4}$, Sumanta Pal ${ }^{5}$, Nizar M. Tannir ${ }^{4}$, Sabina Signoretti ${ }^{6}$, Rajesh Kaldate ${ }^{7 \dagger}$, Christian Scheffold ${ }^{7}$, Evelyn Wang ${ }^{7 \dagger}$, Dana T. Aftab ${ }^{7}$, Bernard Escudier ${ }^{8}$ and Daniel J. George ${ }^{9}$

\begin{abstract}
Background: In the phase 3 METEOR trial, cabozantinib improved progression-free survival (PFS) and overall survival (OS) versus everolimus in patients with advanced RCC after prior antiangiogenic therapy.

Methods: In this exploratory analysis, plasma biomarkers from baseline and week 4 from 621 of 658 randomized patients were analyzed for CA9, HGF, MET, GAS6, AXL, VEGF, VEGFR2, and IL-8. PFS and OS were analyzed by baseline biomarker levels as both dichotomized and continuous variables using univariate and multivariable methods. For on-treatment changes, PFS and OS were analyzed using fold change in biomarker levels at week 4. Biomarkers were considered prognostic if $p<0.05$ and predictive if $p_{\text {interaction }}<0.05$ for the interaction between treatment and biomarker.
\end{abstract}

Results: Hazard ratios for PFS and OS favored cabozantinib versus everolimus for both low and high baseline levels of all biomarkers (hazard ratios $\leq 0.78$ ). In univariate analyses, low baseline HGF, AXL, and VEGF were prognostic for improvements in both PFS and OS with cabozantinib, and low HGF was prognostic for improvements in both PFS and OS with everolimus. Low AXL was predictive of relative improvement in PFS for cabozantinib versus everolimus. Results were generally consistent when baseline biomarkers were expressed as continuous variables, although none were predictive of benefit with treatment. In multivariable analysis, low baseline HGF was independently prognostic for improved PFS for both cabozantinib and everolimus; low HGF, GAS6, and VEGF were independently prognostic for improved OS with cabozantinib. No biomarkers were independently prognostic for OS with everolimus. On-treatment increases in some biomarkers appeared prognostic for PFS or OS with cabozantinib in univariate analyses; however, none were independently prognostic in multivariable analysis.

Conclusions: PFS and OS were improved with cabozantinib versus everolimus at high and low baseline levels of all biomarkers. Low baseline HGF was consistently identified as a prognostic biomarker for improved PFS or OS with cabozantinib or everolimus, supporting further prospective evaluation of the prognostic significance of HGF in advanced RCC.

\footnotetext{
* Correspondence: thomas.powles1@nhs.net

${ }^{\dagger}$ Affiliation at the time of the study.

'Barts Cancer Institute, Queen Mary University of London, London, UK

Full list of author information is available at the end of the article
}

(c) The Author(s). 2021, corrected publication 2021. Open Access This article is licensed under a Creative Commons Attribution 4.0 International License, which permits use, sharing, adaptation, distribution and reproduction in any medium or format, as long as you give appropriate credit to the original author(s) and the source, provide a link to the Creative Commons licence, and indicate if changes were made. The images or other third party material in this article are included in the article's Creative Commons licence, unless indicated otherwise in a credit line to the material. If material is not included in the article's Creative Commons licence and your intended use is not permitted by statutory regulation or exceeds the permitted use, you will need to obtain permission directly from the copyright holder. To view a copy of this licence, visit http://creativecommons.org/ licenses/by/4.0/. The Creative Commons Public Domain Dedication waiver (http://creativecommons.org/publicdomain/zero/1. 0/) applies to the data made available in this article, unless otherwise stated in a credit line to the data. 
Trial registration: ClinicalTrials.gov NCT01865747 (registered on 05/31/2013).

Keywords: Cabozantinib, Everolimus, Renal cell carcinoma, Biomarker, METEOR

\section{Background}

The treatment landscape for advanced renal cell carcinoma (RCC) has vastly expanded in recent years $[1,2]$. VEGF-targeted therapies, mTOR inhibitors, immune checkpoint inhibitors and combination therapies are all standard treatments that have shown improvements in outcome in phase 3 clinical trials. With the growing number of therapies, information on outcomes based on biomarkers may help with optimal therapy selection.

Cabozantinib is a standard of care for the treatment of advanced RCC that has shown efficacy in previouslytreated patients and as a first-line therapy [3-5]. Cabozantinib inhibits multiple tyrosine kinases including MET, AXL, and VEGFR2 [6] that promote oncogenesis, angiogenesis, and resistance to antiangiogenic therapy in RCC. The VHL tumor suppressor gene is frequently inactivated in clear cell RCC, leading to hypoxia and upregulation of hypoxia-controlled genes including VEGF, MET, and AXL [7-10]. VEGF, MET, and AXL have also been associated with poor prognosis in RCC $[9,11,12]$, and MET and AXL have been implicated in resistance to VEGFR-targeted therapy [13].

In the pivotal phase 3 METEOR trial, cabozantinib prolonged progression-free survival (PFS) and overall survival (OS) and increased the objective response rate (ORR) compared with the mTOR inhibitor everolimus in patients with advanced RCC after prior antiangiogenic therapy [3, 4]. Median PFS was 7.4 months with cabozantinib versus 3.9 months with everolimus (HR 0.51, 95\% CI 0.41-0.61, $p<0.0001$ ), and median OS was 21.4 months versus 16.5 months (HR 0.66, 95\% CI 0.53-0.83, $p=0.0003)[3,4]$. Outcomes based on plasma biomarkers was an exploratory endpoint of the METEOR trial. Eight plasma proteins were evaluated for prognostic and predictive significance based on biological relevance and the target profile of cabozantinib: carbonic anhydrase 9 (CA9), hepatocyte growth factor (HGF), MET, GAS6, AXL, VEGF, VEGFR2, and IL-8. To evaluate these potential plasma biomarkers, PFS and OS were analyzed by baseline levels and on-treatment changes for both cabozantinib and everolimus in the METEOR trial.

\section{Methods}

\section{Study design}

Details of the METEOR study design have been published $[3,4]$. The study was registered at ClinicalTrials.gov (NCT01865747, 05/31/2013). The study adhered to the Good Clinical Practice guidelines, the Declaration of Helsinki, and all applicable local laws and regulatory requirements. The study protocol was approved by the institutional review board or ethics committee of all participating centers (the names of the institutional review boards or ethics committees are provided in the Supplement). All patients provided written informed consent.

Patients with advanced RCC with a clear cell component who had been previously treated with up to two prior VEGFR-tyrosine kinase inhibitors (TKIs) were randomized 1:1 to receive cabozantinib (60 $\mathrm{mg}$ daily) or everolimus (10 mg daily). Randomization was stratified by the number of prior VEGFR-TKIs and MSKCC risk group. The primary endpoint was PFS per RECIST 1.1 per independent review committee (IRC), and secondary endpoints were OS and ORR per RECIST 1.1 per IRC. PFS was defined as the time from randomization to radiographic progression or death from any cause. OS was defined as the time from randomization to death from any cause. The relationship of baseline and ontreatment plasma biomarkers with outcomes was an exploratory endpoint.

\section{Assessments and biomarker samples}

Computed tomography $(\mathrm{CT})$ or magnetic resonance imaging (MRI) scans of the chest, abdomen, and pelvis were performed at screening, every 8 weeks for the first 12 months, and every 12 weeks thereafter.

Blood (2 mL) for plasma samples was collected at baseline and at week 4 (week 5 day 1) in K2-EDTA Vacutainer tubes (BD) and processed into plasma within $30 \mathrm{~min}$ by spinning in a refrigerated centrifuge to separate the plasma. Plasma was aliquoted into cryovials and frozen before storing at $-70^{\circ} \mathrm{C}$. Plasma protein levels of CA9, HGF, MET, GAS6, AXL, VEGF, VEGFR2, and IL-8 were measured by ELISA or Luminex assay platforms (Assay Gate, Ijamsville, MD).

\section{Statistical analysis}

Analyses included all patients from the METEOR trial database with available biomarker data. The data cutoff was May 22, 2015 for PFS, and October 31, 2015 for OS.

Baseline biomarkers were considered correlated if $p<$ 0.05 with a Spearman correlation coefficient of $\geq 0.25$ for pairwise interactions. Fold change at week 4 was defined as the biomarker level at week 4 divided by the baseline biomarker level; fold change above or below 1 represents an increase or decrease, respectively, in the biomarker. Baseline and week 4 measurements were paired for each patient to calculate fold change. On-treatment changes in biomarker levels were evaluated for significance $(p<0.05)$ 
using the paired t-test. Association of baseline biomarker levels with IMDC risk group was evaluated by ANOVA using a linear model to test for the difference between group means.

PFS and OS were analyzed using the log-rank test and Cox proportional hazards model. For analyses of baseline biomarkers, PFS and OS were analyzed by subgroups of high vs low biomarker levels dichotomized at the median for each treatment arm. PFS and OS were also analyzed with baseline biomarker levels as a continuous variable, with baseline biomarker levels normalized by $\log _{2}$-transformation in each treatment arm. Analyses of PFS and OS for cabozantinib versus everolimus by subgroups of baseline biomarker levels dichotomized at the median were also conducted. For ontreatment changes, PFS and OS were analyzed using the $\log _{2}$-transformation of the fold change at week 4 as a continuous variable. Biomarkers were considered prognostic for PFS and OS if $p<0.05$ for the analyses. Biomarkers were identified as predictive if the $p$-value for the interaction between treatment and biomarker level ( $\mathrm{p}_{\text {interaction}}$ ) was $<0.05$. Univariate analyses were conducted initially, followed by multivariable analyses that included International Metastatic Renal Cell Carcinoma Database Consortium (IMDC) risk groups (favorable, intermediate, or poor) [14] as cofactors in the model to adjust for differences in baseline risk group status. Separate multivariable analyses that included all biomarkers (baseline and on-treatment changes) were also conducted to determine if biomarkers were independently prognostic; these were run sequentially with baseline biomarkers dichotomized at the median and also with biomarkers expressed as the continuous $\log _{2}$ of baseline levels.

The relationship of baseline and on-treatment plasma biomarkers with outcomes was an exploratory endpoint of the METEOR trial, and the analyses conducted here are retrospective. $P$-values were not adjusted for multiplicity as these are exploratory analyses.

\section{Results}

\section{Patients and baseline plasma biomarker levels and on- treatment changes}

A total of 330 patients were randomized to receive cabozantinib and 328 were randomized to receive everolimus. Demographics and baseline characteristics were generally balanced between treatment arms [3, 4]. Nineteen percent of patients were favorable risk, $64 \%$ were intermediate risk, and $16 \%$ were poor risk according to IMDC prognostic criteria. The majority of patients were male $(75 \%)$ with a median age of 62 years. Plasma samples were available for the majority of patients $(94 \%$ at baseline). Baseline characteristics for patients with biomarker data were similar to those for the randomized population, including for IMDC risk group, sites of metastases, prior therapy and prior nephrectomy (Supplementary Table 1).

Biomarkers evaluated in this study were CA9, HGF, MET, GAS6, AXL, VEGF, VEGFR2, and IL-8. Biomarker levels were measured from collected plasma samples at baseline and week 4 (Table 1). Baseline biomarker data were available for $316 / 330$ patients in the cabozantinib arm and 305/328 patients in the everolimus arm, and fold change data at week 4 were available for 304/330 and 280/328 patients, respectively. Median baseline levels of each of the plasma biomarkers were similar when comparing the cabozantinib and everolimus arms (Table 1). Some of the biomarker levels were found to be correlated at baseline in pairwise analyses (correlation coefficient $\geq 0.25$ and $p<0.05$; Supplementary Figure 1); all biomarker pairs had significant but weak correlations; the highest correlation coefficients $(\sim 0.4)$ were observed

Table 1 Biomarker levels at baseline and week 4

\begin{tabular}{|c|c|c|c|c|c|c|c|c|}
\hline \multirow[t]{3}{*}{$\begin{array}{l}\text { Plasma } \\
\text { Biomarker }\end{array}$} & \multicolumn{4}{|l|}{$\begin{array}{l}\text { Cabozantinib } \\
N=330\end{array}$} & \multicolumn{4}{|l|}{$\begin{array}{l}\text { Everolimus } \\
N=328\end{array}$} \\
\hline & \multirow{2}{*}{$\begin{array}{l}\text { Baseline Level } \\
\text { Median }\end{array}$} & \multicolumn{2}{|c|}{ Fold Change at week 4} & \multirow[t]{2}{*}{$p$-value } & \multirow{2}{*}{$\begin{array}{l}\text { Baseline Level } \\
\text { Median }\end{array}$} & \multicolumn{2}{|c|}{ Fold Change at week 4} & \multirow[t]{2}{*}{$p$-value } \\
\hline & & Median & Mean & & & Median & Mean & \\
\hline CA9 & 77.92 & 1.93 & 4.84 & $<0.0001$ & 87.49 & 1.15 & 2.60 & $<0.0001$ \\
\hline HGF & 745.0 & 0.86 & 1.09 & $<0.0001$ & 721.1 & 1.09 & 1.51 & 0.002 \\
\hline MET & 182.8 & 1.09 & 1.13 & $<0.0001$ & 178.0 & 0.98 & 1.00 & 0.05 \\
\hline GAS6 & 14,760 & 1.40 & 1.51 & $<0.0001$ & 14,570 & 1.12 & 1.11 & $<0.0001$ \\
\hline$A X L$ & 14,820 & 1.14 & 1.20 & $<0.0001$ & 14,690 & 0.92 & 0.94 & $<0.0001$ \\
\hline VEGF & 11.55 & 2.57 & 6.74 & $<0.0001$ & 11.20 & 1.11 & 3.65 & 0.005 \\
\hline VEGFR2 & 4699 & 0.79 & 0.85 & $<0.0001$ & 4918 & 0.84 & 0.89 & $<0.0001$ \\
\hline IL-8 & 4.601 & 1.23 & 3.44 & 0.004 & 5.049 & 1.32 & 6.46 & $<0.0001$ \\
\hline
\end{tabular}

All values in $\mathrm{pg} / \mathrm{mL}$ except for MET which is in $\mathrm{ng} / \mathrm{mL}$

Plasma biomarker baseline and fold change data were available for 316 and 304 patients in the cabozantinib arm and 305 and 280 patients in the everolimus arm, respectively, with the exception of IL- 8 in the everolimus arm, for which 304 and 279 patients had available data, respectively 
between AXL and GAS6, VEGF and IL8, and MET and GAS6. An ANOVA was performed to assess the association between baseline biomarker levels and IMDC risk groups. All biomarker levels were significantly different between IMDC risk groups except for CA9, with the largest variance observed for HGF and VEGF between IMDC groups (Supplementary Table 2). For all biomarkers, mean levels were highest in the poor risk group, and for most (HGF, MET, GAS6, AXL, VEGF, and IL-8) relative levels were poor $>$ intermediate $>$ favorable; for VEGFR2, levels were poor $>$ favorable>intermediate.

At week 4, all biomarkers had significant changes from baseline in both treatment arms (Table 1), with most biomarker levels increasing (fold change> 1). In the cabozantinib arm, mean biomarker levels of CA9, HGF, MET, GAS6, VEGF, and IL-8 increased, and mean levels of VEGFR2 decreased (fold change $<1$ ). In the everolimus arm, mean levels of CA9, HGF, GAS6, VEGF, and IL-8 increased, and mean levels of VEGFR2 decreased. Biomarkers with the largest increases at week 4 were VEGF (mean fold change of 6.7 with cabozantinib and 3.6 with everolimus), CA9 (4.8 and 2.6), and IL-8 (3.4 and 6.5); all other biomarkers had mean fold increases of $\leq 1.5$ in both treatment arms.

\section{Progression-free survival and overall survival by baseline biomarker levels}

PFS and OS were analyzed by baseline biomarker levels ( $\geq$ median vs $<$ median) for each treatment arm. Results for PFS are shown in Table 2. Low vs high baseline levels of HGF, AXL, and VEGF were associated with longer PFS with cabozantinib $(p<0.05)$, and low vs high baseline levels of HGF and IL- 8 were associated with longer PFS with everolimus. Kaplan-Meier plots of PFS by high and low biomarker levels are shown for HGF, AXL, and VEGF in Fig. 1.

Analyses of PFS for cabozantinib versus everolimus were also conducted by subgroups of high and low biomarker level. Cabozantinib was associated with prolonged PFS compared with everolimus for both high and low levels of all biomarkers analyzed with all HRs $\leq 0.65$ (Supplementary Table 3). Low levels of AXL were predictive for an improved relative PFS benefit with cabozantinib compared with everolimus (Table 2).

Analysis of OS by baseline biomarkers ( $\geq$ median vs $<$ median) for each treatment arm is shown in Table 3. Low vs high levels of HGF, GAS6, AXL, VEGF, and IL-8 were associated with longer OS with cabozantinib, and low vs high levels of HGF, MET, GAS6, VEGF, and IL-8 were associated with longer OS with everolimus $(p<$ $0.05)$. Kaplan-Meier plots of OS by high and low biomarker levels are shown for HGF, AXL, and VEGF in Fig. 1.

Analyses of OS for cabozantinib versus everolimus were also conducted by subgroups of low and high biomarker level. Cabozantinib was associated with prolonged OS compared with everolimus $(\mathrm{HR}<1)$ for both low and high levels of all biomarkers analyzed with all HRs $\leq 0.78$ (Supplementary Table 3). None of the biomarkers were predictive for a differential treatment effect at a significance level of 0.05; HGF, GAS6, AXL, and VEGF had the lowest $p_{\text {interaction }}$ values $\left(p_{\text {interaction }}<0.15\right)$ (Table 3).

PFS and OS were also analyzed with the $\log _{2}$ of baseline biomarker levels as continuous variables. In general, the analyses gave similar results to those using

Table 2 Progression-free survival within each treatment arm by baseline biomarker levels dichotomized at the median

\begin{tabular}{|c|c|c|c|c|c|c|c|}
\hline \multirow{3}{*}{$\begin{array}{l}\text { Plasma } \\
\text { Biomarker }\end{array}$} & \multicolumn{3}{|c|}{ Cabozantinib } & \multicolumn{3}{|l|}{ Everolimus } & \multirow[t]{3}{*}{$P_{\text {interaction }}$} \\
\hline & \multicolumn{2}{|c|}{ Median PFS, mo } & \multirow[t]{2}{*}{ HR $(95 \% \mathrm{Cl})$} & \multicolumn{2}{|c|}{ Median PFS, mo } & \multirow[t]{2}{*}{ HR $(95 \% \mathrm{Cl})$} & \\
\hline & $\begin{array}{l}\geq \text { median } \\
\text { biomarker }\end{array}$ & $\begin{array}{l}<\text { median } \\
\text { biomarker }\end{array}$ & & $\begin{array}{l}\geq \text { median } \\
\text { biomarker }\end{array}$ & $\begin{array}{l}<\text { median } \\
\text { biomarker }\end{array}$ & & \\
\hline CA9 & 7.4 & 7.4 & $0.87(0.65,1.17)$ & 3.9 & 3.8 & $0.89(0.67,1.17)$ & 1.0 \\
\hline HGF & 5.6 & 9.2 & $1.77(1.30,2.40)^{*}$ & 3.7 & 5.4 & $1.48(1.12,1.95)^{*}$ & 0.47 \\
\hline MET & 7.4 & 7.4 & $1.00(0.74,1.35)$ & 3.7 & 4.1 & $1.16(0.88,1.53)$ & 0.45 \\
\hline GAS6 & 7.2 & 9.1 & $1.31(0.97,1.77)$ & 3.7 & 3.9 & $1.07(0.81,1.40)$ & 0.36 \\
\hline$A X L$ & 6.0 & 9.1 & $1.45(1.07,1.96)^{*}$ & 3.8 & 3.9 & $0.92(0.70,1.21)$ & $0.02^{*}$ \\
\hline VEGF & 5.6 & 9.2 & $1.57(1.17,2.12)^{*}$ & 3.7 & 4.1 & $1.15(0.87,1.51)$ & 0.16 \\
\hline VEGFR2 & 7.4 & 7.4 & $0.89(0.66,1.19)$ & 4.4 & 3.7 & $0.88(0.67,1.16)$ & 0.94 \\
\hline IL-8 & 7.3 & 7.4 & $1.10(0.82,1.49)$ & 3.7 & 5.3 & $1.40(1.06,1.85)^{*}$ & 0.21 \\
\hline
\end{tabular}

Hazard ratios are for high ( $\geq$ median) versus low ( $<$ median) biomarker levels

P-interaction was obtained from a separate model that included the interaction between treatment and biomarker level

${ }^{*} p<0.05$ for the analysis 

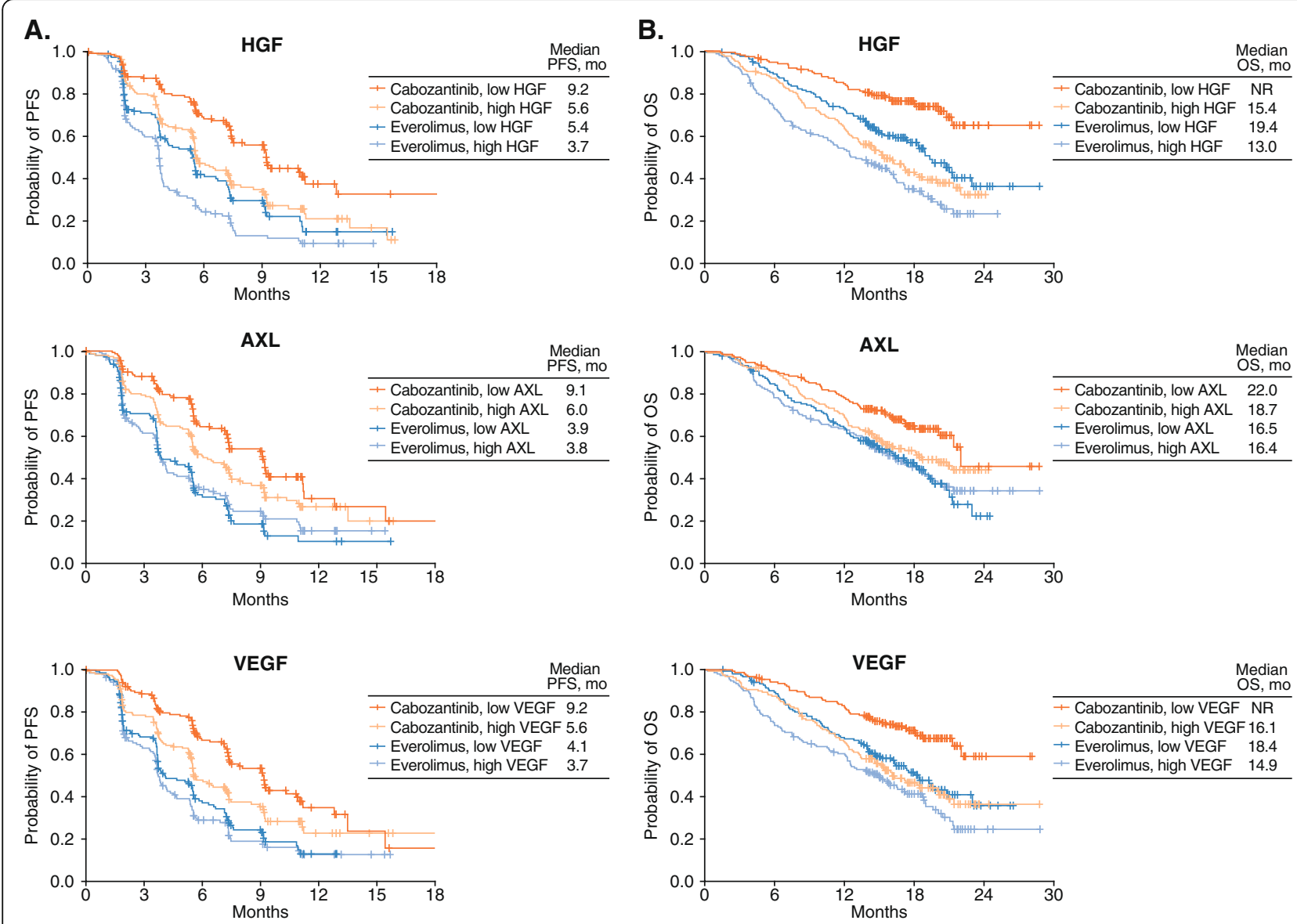

High levels and low levels are defined by $\geq$ median and $<$ median, respectively.

NR, not reached

Fig. 1 Kaplan-Meier plot of progression-free survival (A) and overall survival (B) by baseline biomarker levels dichotomized at the median

Table 3 Overall survival within each treatment arm by baseline biomarker levels dichotomized at the median

\begin{tabular}{|c|c|c|c|c|c|c|c|}
\hline \multirow{4}{*}{$\begin{array}{l}\text { Plasma } \\
\text { Biomarker }\end{array}$} & \multicolumn{6}{|c|}{ Overall Survival } & \multirow[t]{4}{*}{$P_{\text {interaction }}$} \\
\hline & \multicolumn{3}{|c|}{ Cabozantinib } & \multicolumn{3}{|l|}{ Everolimus } & \\
\hline & \multicolumn{2}{|c|}{ Median OS, mo } & \multirow[t]{2}{*}{ HR (95\% Cl) } & \multicolumn{2}{|l|}{ Median OS, mo } & \multirow[t]{2}{*}{ HR $(95 \% \mathrm{Cl})$} & \\
\hline & $\begin{array}{l}\geq \text { median } \\
\text { biomarker }\end{array}$ & $<$ median biomarker & & $\geq$ median biomarker & $<$ median biomarker & & \\
\hline CA9 & 21.4 & 22.0 & $1.19(0.85,1.68)$ & 15.4 & 16.5 & $1.20(0.89,1.62)$ & 0.99 \\
\hline HGF & 15.4 & $N R$ & $2.79(1.92,4.05)^{*}$ & 13.0 & 19.4 & $1.78(1.32,2.42)^{*}$ & 0.08 \\
\hline MET & 19.9 & NR & $1.29(0.91,1.81)$ & 15.0 & 18.9 & $1.42(1.05,1.91)^{*}$ & 0.70 \\
\hline GAS6 & 17.2 & NR & $2.01(1.41,2.86)^{*}$ & 13.9 & 18.4 & $1.42(1.05,1.92)^{*}$ & 0.14 \\
\hline$A X L$ & 18.7 & 22.0 & $1.48(1.05,2.09)^{*}$ & 16.4 & 16.5 & $1.00(0.74,1.35)$ & 0.11 \\
\hline VEGF & 16.1 & NR & $2.16(1.51,3.08)^{*}$ & 14.9 & 18.4 & $1.43(1.06,1.94)^{*}$ & 0.09 \\
\hline VEGFR2 & 21.4 & 22.0 & $0.97(0.69,1.36)$ & 16.5 & 16.4 & $0.90(0.66,1.21)$ & 0.75 \\
\hline IL-8 & 17.2 & NR & $1.90(1.34,2.68)^{*}$ & 13.0 & 19.4 & $1.78(1.31,2.42)^{*}$ & 0.73 \\
\hline
\end{tabular}

Hazard ratios are for high ( $\geq$ median) versus low (<median) biomarker levels. P-interaction was obtained from a separate model that included the interaction between treatment and biomarker level

$N R$ not reached

${ }^{*} p<0.05$ for the analysis 
dichotomized levels, with decreasing levels of some biomarkers associated with improvements in PFS or OS (Supplementary Table 4). In the continuous analyses, additional biomarkers identified as prognostic for improved PFS were decreased levels of MET with cabozantinib, and additional biomarkers prognostic for improved OS were decreased levels of MET with cabozantinib and decreased levels of AXL and CA9 with everolimus. No biomarkers were predictive in analyses with baseline biomarker levels expressed as continuous variables; AXL had the lowest p-interaction value for PFS and GAS6 had the lowest $\mathrm{p}$-interaction for OS ( $\mathrm{p}_{\text {interaction }}$ values $<0.15$ ).

\section{Multivariable analyses of baseline biomarkers adjusting for IMDC risk group}

Baseline biomarkers dichotomized at the median were further investigated by multivariable analyses including IMDC risk group as a covariate in the model; results are shown in Table 4. Each biomarker was adjusted with IMDC risk group in separate analyses. All biomarkers identified as prognostic for PFS in the univariate analyses were independently prognostic when adjusting for IMDC risk group; low vs high baseline levels of HGF, AXL, and VEGF remained prognostic for improved PFS with cabozantinib, and low vs high baseline levels of HGF and IL-8 remained prognostic for improved PFS with everolimus. Low levels of AXL remained predictive for an improved relative PFS benefit with cabozantinib compared with everolimus. In multivariable analyses of OS adjusting for IMDC risk group, low vs high baseline levels of HGF, GAS6, VEGF, and IL-8 were independently prognostic for OS with cabozantinib. For everolimus, HGF, GAS6, and IL-8 were independently prognostic for OS.

Baseline biomarkers expressed as continuous variables were also run in multivariable analyses including IMDC risk group as a covariate in the model (Supplementary Table 5). Decreasing levels of baseline HGF and AXL were independently prognostic for improved PFS with cabozantinib and decreasing levels of baseline HGF were independently prognostic for improved PFS with everolimus. For OS, decreasing baseline levels of HGF, MET, GAS6, VEGF, and IL-8 were independently prognostic for cabozantinib, and decreasing baseline levels of HGF, MET, GAS6, VEGF, and IL-8 were independently prognostic with everolimus. No biomarkers were predictive for an improved relative PFS or OS benefit for cabozantinib or everolimus at a significance level of 0.05 . AXL had the lowest pinteraction values for PFS and GAS6 had the lowest $\mathrm{p}_{\text {interaction }}$ value for OS ( $\mathrm{p}_{\text {interaction }}$ values $<0.15$ ).

\section{PFS and OS analyzed by continuous on-treatment changes in biomarker levels}

Univariate analyses of PFS and OS based on the $\log _{2}$ transformation of fold change at week 4 as a continuous variable are shown in Table 5. In analyses of PFS, an increase in HGF at week 4 was associated with improved PFS in the cabozantinib group. An increase in HGF was also predictive for relative improvement in PFS for cabozantinib compared with everolimus ( $p_{\text {interaction }}=0.02$ ).

In analyses of OS, an increase in HGF, VEGF, or IL-8 at week 4 was associated with improved OS in the cabozantinib group (Table 5). For the everolimus group, none of the on-treatment changes in biomarker level were associated with OS. No biomarkers were predictive for a differential treatment effect; the lowest pinteraction values ( $\mathrm{p}_{\text {interaction }}<0.15$ ) were for HGF and GAS6.

\section{Multivariable analyses of PFS and OS with multiple biomarkers}

Biomarkers were further investigated by multivariable analyses including all biomarkers which had $p<0.10$ in the univariate analyses. Multivariable analyses were run separately for baseline biomarkers dichotomized at the median (Table 6) and baseline biomarkers as continuous variables (Supplementary Table 6) with each analysis

Table 4 Multivariable analyses of PFS and OS including IMDC risk group in each treatment arm by baseline biomarker level dichotomized at the median

\begin{tabular}{|c|c|c|c|c|c|c|}
\hline \multirow[t]{2}{*}{$\begin{array}{l}\text { Plasma } \\
\text { Biomarker }\end{array}$} & \multicolumn{3}{|c|}{$\begin{array}{l}\text { Progression-Free Survival } \\
\text { HR }(95 \% \mathrm{Cl})\end{array}$} & \multicolumn{3}{|l|}{$\begin{array}{l}\text { Overall Survival } \\
\text { HR }(95 \% \mathrm{Cl})\end{array}$} \\
\hline & Cabozantinib & Everolimus & $P_{\text {interaction }}$ & Cabozantinib & Everolimus & $\overline{P_{\text {interaction }}}$ \\
\hline$\overline{H G F}$ & $1.57(1.14,2.16)^{*}$ & $1.39(1.05,1.84)^{*}$ & 0.53 & $2.28(1.55,3.35)^{*}$ & $1.57(1.16,2.13)^{*}$ & 0.16 \\
\hline MET & $0.96(0.71,1.29)$ & $1.11(0.84,1.47)$ & 0.57 & $1.24(0.88,1.74)$ & $1.33(0.98,1.8)$ & 0.80 \\
\hline GAS6 & $1.29(0.96,1.74)$ & $1.05(0.80,1.39)$ & 0.35 & $1.83(1.28,2.6)^{*}$ & $1.37(1.01,1.86)^{*}$ & 0.20 \\
\hline$A X L$ & $1.39(1.03,1.88)^{*}$ & $0.87(0.65,1.15)$ & $0.013^{*}$ & $1.40(0.99,1.98)$ & $0.98(0.72,1.32)$ & 0.14 \\
\hline VEGF & $1.41(1.03,1.94)^{*}$ & $1.07(0.81,1.42)$ & 0.16 & $1.72(1.19,2.49)^{*}$ & $1.34(0.98,1.82)$ & 0.29 \\
\hline IL-8 & $1.03(0.76,1.40)$ & $1.33(1.01,1.76)^{*}$ & 0.24 & $1.77(1.25,2.50)^{*}$ & $1.67(1.23,2.27)^{*}$ & 0.76 \\
\hline
\end{tabular}

Hazard ratios are for high ( $\geq$ median) versus low ( $<$ median) biomarker levels. IMDC risk groups were included as cofactors in the multivariable analysis. Biomarkers were included in the multivariable analysis that had $p<0.1$ in at least one of the univariate analyses for PFS or OS by treatment arm. P-interaction was obtained from a separate model that included the interaction between treatment and biomarker level

${ }^{*} p<0.05$ for the analysis 
Table 5 Univariate analyses of progression-free survival and overall survival based on continuous $\log _{2}$ fold change in biomarkers at week 4

\begin{tabular}{|c|c|c|c|c|c|c|}
\hline \multirow{2}{*}{$\begin{array}{l}\text { Plasma } \\
\text { Biomarker }\end{array}$} & \multicolumn{2}{|c|}{ Hazard Ratio $(95 \% \mathrm{Cl})$ for PFS } & \multirow[t]{2}{*}{$P_{\text {interaction }}$} & \multicolumn{2}{|c|}{ Hazard Ratio $(95 \% \mathrm{Cl})$ for OS } & \multirow[t]{2}{*}{$P_{\text {interaction }}$} \\
\hline & Cabozantinib & Everolimus & & Cabozantinib & Everolimus & \\
\hline CA9 & $1.00(0.92,1.08)$ & $1.04(0.95,1.14)$ & 0.48 & $0.92(0.84,1.00)$ & $0.93(0.83,1.03)$ & 0.86 \\
\hline HGF & $0.77(0.63,0.93)^{*}$ & $1.06(0.88,1.28)$ & $0.02^{*}$ & $0.80(0.65,0.98)^{*}$ & $1.00(0.80,1.26)$ & 0.14 \\
\hline MET & $1.16(0.74,1.83)$ & $0.70(0.41,1.19)$ & 0.13 & $1.02(0.62,1.67)$ & $0.79(0.43,1.42)$ & 0.52 \\
\hline GAS6 & $0.87(0.60,1.28)$ & $0.88(0.61,1.29)$ & 0.92 & $0.67(0.44,1.01)$ & $1.05(0.68,1.60)$ & 0.13 \\
\hline AXL & $0.67(0.41,1.11)$ & $0.88(0.54,1.44)$ & 0.53 & $0.70(0.41,1.19)$ & $0.86(0.51,1.47)$ & 0.55 \\
\hline VEGF & $0.95(0.89,1.01)$ & $1.03(0.95,1.11)$ & 0.13 & $0.90(0.84,0.97)^{*}$ & $0.92(0.85,1.01)$ & 0.63 \\
\hline VEGFR2 & $1.05(0.69,1.60)$ & $0.86(0.54,1.34)$ & 0.52 & $1.06(0.70,1.61)$ & $0.86(0.53,1.42)$ & 0.48 \\
\hline IL-8 & $0.93(0.85,1.01)$ & $1.00(0.92,1.09)$ & 0.26 & $0.91(0.83,1.00)^{*}$ & $0.96(0.88,1.06)$ & 0.37 \\
\hline
\end{tabular}

P-interaction was obtained from a separate model that included the interaction between treatment and biomarker level

${ }^{*} p<0.05$ for the analysis

including on-treatment changes in biomarkers as covariates. For analyses including baseline biomarkers dichotomized at the median, low vs high levels of HGF were independently prognostic for improved PFS for both cabozantinib and everolimus. For OS, low vs high levels of HGF, GAS6, and VEGF were independently prognostic for OS with cabozantinib; no biomarkers were independently prognostic for OS with everolimus.

For analyses including baseline biomarkers expressed as continuous variables, decreasing levels of AXL were independently prognostic for improved PFS with cabozantinib and decreasing levels of HGF were prognostic for improved PFS with everolimus. For OS, decreasing levels of HGF and GAS6 were both independently prognostic for improved OS with cabozantinib and decreasing levels of HGF were independently prognostic for improved OS with everolimus.

No on-treatment changes in biomarkers were independently prognostic for PFS or OS in any of the multivariable analyses (Table 6 and Supplementary Table 6).

\section{Discussion}

The METEOR trial showed that cabozantinib improved PFS, OS, and ORR compared with everolimus in patients with advanced RCC who received prior antiangiogenic therapy. In the current study, PFS and OS were analyzed based on eight plasma biomarkers to test for prognostic and predictive significance: CA9, HGF, MET, GAS6, AXL, VEGF, VEGFR2, and IL-8. These biomarkers were selected based on biological relevance to renal cell carcinoma, previous reports of prognostic significance, and the target profile of cabozantinib including both cabozantinib receptor targets (VEGFR2, MET, and AXL) and their ligands (VEGF, HGF, and GAS6). CA9 and IL-8 were selected based on previous reports of prognostic significance in RCC [15-17].

PFS and OS favored cabozantinib versus everolimus for both low and high baseline levels of all biomarkers analyzed (hazard ratios $\leq 0.78$ ), suggesting a benefit with cabozantinib treatment irrespective of biomarker status. In univariate analyses of the cabozantinib arm based on high vs low baseline biomarker levels, low HGF, AXL, and VEGF were prognostic for improved PFS and low HGF, GAS6, AXL, VEGF, and IL-8 were prognostic for improved OS. In the everolimus arm, low HGF and IL-8 were prognostic for improved PFS and low HGF, MET, GAS6, VEGF, and IL-8 were prognostic for improved OS. Results with baseline biomarkers expressed as continuous variables were generally consistent.

Multivariable analyses were performed to determine if biomarkers were independently prognostic for outcome. In analyses adjusting for IMDC risk group, low HGF was independently prognostic for improved PFS and OS, and low GAS6 and IL-8 were independently prognostic for improved OS, for both cabozantinib and everolimus. Low VEGF was independently prognostic for improved OS for cabozantinib only. Baseline levels of all biomarkers were significantly different between IMDC risk groups except for CA9, suggesting an association of biomarker level with risk status, which has been previously reported for VEGF and MSKCC risk status [18]. Separate multivariable analyses for outcome including all baseline biomarkers were performed to test for the independence of baseline biomarkers with respect to each other. In these analyses low baseline HGF was independently prognostic for improved PFS for both cabozantinib and everolimus in most of the analyses, although it did not meet the threshold for prognostic significance with cabozantinib in analyses based on continuous biomarker level. Low baseline HGF and GAS6 were independently prognostic for improved OS with cabozantinib in all of the analyses.

Low HGF, VEGF, and IL-8 have all been implicated as prognostic biomarkers for improved PFS or OS in previous studies of RCC with targeted therapy, interferon- $\alpha$, or placebo [17-20]. GAS6 and its cognate receptor AXL 
Table 6 Multivariable analyses of progression-free survival and overall survival in each treatment arm including baseline biomarkers dichotomized at the median and change in biomarkers at week 4 as covariates

\begin{tabular}{|c|c|c|}
\hline Plasma Biomarker & $\mathrm{HR}(95 \% \mathrm{Cl})$ & $P_{\text {value }}$ \\
\hline \multicolumn{3}{|c|}{ Progression-Free Survival (Cabozantinib) } \\
\hline HGF & $1.46(1.02,2.08)$ & $0.04^{*}$ \\
\hline VEGF & $1.32(0.95,1.83)$ & 0.10 \\
\hline$A X L$ & $1.31(0.95,1.79)$ & 0.10 \\
\hline$\triangle H G F$ & $0.87(0.70,1.07)$ & 0.19 \\
\hline GAS6 & $1.08(0.79,1.49)$ & 0.62 \\
\hline$\Delta \mathrm{L}-8$ & $0.98(0.90,1.07)$ & 0.68 \\
\hline \multicolumn{3}{|c|}{ Overall Survival (Cabozantinib) } \\
\hline HGF & $2.17(1.41,3.35)$ & $<0.001^{*}$ \\
\hline GAS6 & $1.62(1.08-2.42)$ & $0.02^{*}$ \\
\hline VEGF & $1.62(1.04,2.53)$ & $0.03^{*}$ \\
\hline$A X L$ & $1.19(0.83,1.72)$ & 0.34 \\
\hline IL-8 & $1.15(0.76,1.76)$ & 0.51 \\
\hline$\triangle C A 9$ & $0.97(0.87,1.07)$ & 0.52 \\
\hline$\triangle \mathrm{VEGF}$ & $1.02(0.93,1.11)$ & 0.74 \\
\hline$\triangle H G F$ & $0.98(0.77,1.23)$ & 0.85 \\
\hline$\triangle \mathrm{GAS6}$ & $0.98(0.6,1.59)$ & 0.93 \\
\hline$\Delta \mathrm{L}-8$ & $1(0.89,1.13)$ & 0.94 \\
\hline
\end{tabular}

Progression-Free Survival (Everolimus)

$\begin{array}{lll}\text { HGF } & 1.37(1.03,1.82) & 0.03^{*} \\ \text { IL-8 } & 1.29(0.97,1.71) & 0.08\end{array}$

Overall Survival (Everolimus)

$\begin{array}{lll}\text { MET } & 1.37(0.99,1.90) & 0.06 \\ \text { HGF } & 1.38(0.99,1.93) & 0.06 \\ \text { IL-8 } & 1.35(0.95,1.91) & 0.09 \\ \text { GAS6 } & 1.20(0.86,1.67) & 0.28 \\ \text { DVEGF } & 0.96(0.87,1.06) & 0.46 \\ \text { VEGF } & 1.07(0.74,1.55) & 0.72\end{array}$

Biomarkers were included in the multivariable analysis if $p<0.10$ in the univariate analyses. Hazard ratios are for high versus low biomarker levels. $\Delta$ indicates the covariate is change in the biomarker at week 4; all other covariates are baseline biomarkers dichotomized at the median ${ }^{*} p<0.05$ for the analysis

have not been extensively studied as biomarkers, but higher levels of expression of both GAS6 and AXL have been reported to be associated with worse survival [12]. That low levels of both HGF and GAS6 demonstrated prognostic significance with cabozantinib treatment across multiple analyses is relevant for several reasons. First, these biomarkers are ligands for two of the key targets inhibited by cabozantinib (MET and AXL), and these targets differentiate cabozantinib from other TKIs. In the current study, patients with low levels of HGF had a more favorable prognosis for PFS and OS in both treatment arms, although there was a numerically greater improvement for cabozantinib compared with everolimus in many of the analyses. The results presented here show that HGF and GAS6 have prognostic significance for cabozantinib monotherapy; however, we hypothesize that these biomarkers could also have prognostic significance for cabozantinib in combination with other agents including checkpoint inhibitors. The RCC treatment landscape is evolving, and cabozantinib has recently been approved in combination with nivolumab for patients with previously untreated advanced RCC [21]. Biomarker studies of cabozantinib plus nivolumab and other therapies should evaluate HGF and GAS6 levels to further assess their significance as prognostic and predictive biomarkers in RCC.

Pharmacodynamic changes for cabozantinib targets were consistent with previous reports with cabozantinib treatment, with all biomarkers increasing except for VEGFR2, which decreased in both arms [22, 23]. The largest on-treatment increases were observed for VEGF, CA9, and IL-8 in both treatment arms. Increases in VEGF and CA9 and decreases in VEGFR2 with cabozantinib treatment have been observed in triple negative breast cancer [22], and increases in VEGF, CA9, MET, and IL- 8 and decreases in VEGFR2 with cabozantinib treatment have been observed in castration-resistant prostate cancer [23]. Increases in VEGF and decreases in VEGFR2 with other TKIs that inhibit VEGFR2, including sunitinib and sorafenib, have also been observed in RCC $[17,20,24]$. Although on-treatment changes in some biomarkers (HGF, VEGF, IL-8) appeared prognostic for improved PFS or OS with cabozantinib in univariate analyses, none of these were independently prognostic in multivariable analyses.

The METEOR trial was not powered to evaluate outcomes based on biomarkers, which was an exploratory endpoint. Nonetheless, a large number of patients in this phase 3 trial had data available for the analyses. The current study identified several potential prognostic baseline biomarkers; however, the lack of a placebo arm limits the ability to separate the prognostic effect from the treatment effect. The analyses presented here focused on plasma biomarkers because of the relative ease of obtaining contemporaneous samples from patients. Tumor MET status from available archival or recently biopsied tumor tissue has also been analyzed in the METEOR trial and was not found to be predictive of benefit with cabozantinib [25].

No plasma biomarkers were found to be consistently predictive for an improved benefit in the analyses; however, low baseline levels of AXL were predictive for improved PFS with cabozantinib in some of the analyses. Evaluation of interaction terms for predictive biomarkers requires anywhere from 4 to 16 times the sample size needed for testing main effects, and one approach to 
address this is to raise the cutoff for the $p$-value when considering interactions [26]. Clear predictive biomarkers for treatment of RCC have not been reported [27], and some studies have taken the approach of using multiple biomarkers to calculate a composite biomarker score for benefit, including for everolimus versus sunitinib [19] and more recently, for lenvatinib plus everolimus versus everolimus alone [28]. Additional studies are needed to identify predictive biomarkers in advanced RCC.

\section{Conclusions}

In the METEOR trial, multiple baseline plasma biomarkers were prognostic for PFS or OS with cabozantinib or everolimus treatment. In particular, low baseline levels of HGF and GAS6, cognate ligands for MET and AXL, were prognostic for improved PFS or OS with cabozantinib treatment, supporting further studies of these biomarkers in patients with advanced RCC, where several TKI-immuno-oncology combinations, including cabozantinib and nivolumab, have demonstrated clinical benefit.

\section{Abbreviations}

$\mathrm{CT}$ : Computed tomography; IMDC: International Metastatic Renal Cell Carcinoma Database Consortium; IRC: Independent review committee; MRI: Magnetic resonance imaging; ORR: Objective response rate; OS: Overall survival; PFS: Progression-free survival; RCC: Renal cell carcinoma;

TKl: Tyrosine kinase inhibitor

\section{Supplementary Information}

The online version contains supplementary material available at https://doi. org/10.1186/s12885-021-08630-w.

\section{Additional file 1.}

\section{Acknowledgements}

The authors would like to thank the patients who have participated in the METEOR study, the investigators, nursing staff, and research support staff in participating institutions, under whom this multi-site trial was conducted. Patients treated at Memorial Sloan Kettering Cancer Center were supported in part by Memorial Sloan Kettering Cancer Center Support Grant/Core Grant (P30 CA008748). The authors would also like to thank Douglas Laird for his contributions to the design and initial execution of the METEOR biomarker program. Statistical analysis was performed by Fios Genomics Ltd. Medical writing support was provided by Julie C Lougheed, PhD (Exelixis) with editorial assistance by Fishawack Communications (Conshohocken, PA, USA), which was funded by Exelixis.

\section{Authors' contributions}

TP, TKC, and DJG contributed to the study conception and design. TP, TKC, RJM, EJ, NMT, BE, and DJG contributed to data acquisition. RK provided data analyses. All authors contributed to the interpretation of data. TP, TC, and DJG contributed to the drafting of the manuscript. All authors reviewed and approved the final manuscript.

\section{Funding}

The study was sponsored and funded by Exelixis, Inc. (Alameda, CA). The funder was involved in the study design, data collection, and analysis.

\section{Availability of data and materials}

The datasets used and/or analyzed during the current study are available from the corresponding author or sponsor author on reasonable request.

\section{Declarations}

\section{Ethics approval and consent to participate}

The study adhered to the Good Clinical Practice guidelines, the Declaration of Helsinki, and all applicable local laws and regulatory requirements. The study protocol was approved by the institutional review board or ethics committee of all participating centers (the names of the institutional review boards or ethics committees are provided in the Supplement). All patients provided written informed consent.

\section{Consent for publication}

Not applicable.

\section{Competing interests}

Dr. Powles has received honoraria from Astellas Pharma, AstraZeneca, BristolMyers Squibb, Eisai, Exelixis, Incyte, Ipsen, Johnson \& Johnson, Merck, Merck Serono, MSD, Novartis, Pfizer, Roche, and Seattle Genetics; has a consulting or advisory role with Astellas Pharma, AstraZeneca, Bristol-Myers Squibb, Eisai, Exelixis, Incyte, Ipsen, Johnson \& Johnson, Merck, Merck Serono, MSD, Novartis, Pfizer, Roche, and Seattle Genetics; has received research funding from Astellas Pharma, AstraZeneca, Bristol-Myers Squibb, Eisai, Exelixis, Ipsen, Johnson \& Johnson, Merck, Merck Serono, MSD, Novartis, Pfizer, Roche, and Seattle Genetics; and has received travel/accommodation/other expenses from AstraZeneca, Ipsen, MSD, Pfizer, and Roche.

Dr. Choueiri has consulting relationships with AstraZeneca, Bristol-Myers Squibb, Eisai, Eli Lilly and Company, EMD Serono, Exelixis, GlaxoSmithKline, Merck, Novartis, and Pfizer; has received institutional research grants from Bristol-Myers Squibb, Exelixis, and GlaxoSmithKline; has received travel fees from Bristol-Myers Squibb, Eisai, Eli Lilly and Company, Exelixis, Merck, Novartis, and Pfizer; and has stock in Pionyr and Tempest.

Dr. Motzer has received grants from Eisai, Pfizer, Novartis, Exelixis, Genentech, Roche, and Bristol Myers Squibb; and has received personal fees from Eisai, Merck, Pfizer, Novartis, Exelixis, Genentech, Incyte, Lilly, Roche, Bristol Myers Squibb, Astra Zeneca, and EMD Serono.

Dr. Jonasch has received grants from Exelixis, Aravive, Merck, and Novartis; and has received personal fees from Exelixis, Aravive, Eisai, Calithera, Merck, Novartis, and Pfizer.

Dr. Pal has a consulting or advisory role with Astellas Pharma, Aveo, BristolMyers Squibb, Eisai, Exelixis, Genentech, Ipsen, Myriad Pharmaceuticals, Novartis, and Pfizer; and has received research funding from Medivation. Dr. Tannir has a consulting or advisory role with Bristol-Myers-Squibb, Pfizer, Nektar Therapeutics, Exelixis, Inc., Eisai Medical Research, Eli Lilly, Oncorena, Ono Pharmaceutical, Calithera Bioscience, Surface Oncology, Novartis, Ipsen, and Merck Sharp \& Dohme; has received research funding from BristolMyers-Squibb, Pfizer, Nektar Therapeutics, Exelixis, Inc., Calithera Bioscience, Mirati Therapeutics, Epizyme Inc., Lilly, Arrowhead Pharmaceuticals, Inc., Takeda, and Eisai; and is on scientific advisory committees with Nektar Therapeutics, Pfizer, Oncorena, Eli Lilly, and Eisai Medical Research.

Dr. Signoretti has a consulting or advisory role with AstraZeneca/ Medlmmune, Bristol-Myers Squibb, and Merck; has received institutional research funding from AstraZeneca, Bristol-Myers Squibb, Exelixis, and Novartis; has received royalties from Biogenex.

Dr. Kaldate is a former employee of Exelixis; has stock/ownership interests with Exelixis.

Dr. Scheffold is employed by Exelixis; has stock/ownership interests with Exelixis; and has a patent.

Dr. Wang is a former employee of Exelixis; has stock/ownership interests with Exelixis.

Dr. Aftab is employed by Exelixis; has stock/ownership interests with Exelixis; and has a patent.

Dr. Escudier has received grants from BMS, Novartis, and Aveo; and has received personal fees from BMS, Aveo, Ipsen, Pfizer, Oncorena, Immunicum, Novartis, and Roche

Dr. George is a senior editor for American Association of Cancer Research; is a co-editor-in-chief for Clin Adv Hematol Oncol (Millennium Medical Publishing); has a consulting or advisory role with Astellas, AstraZeneca, Bayer Pharmaceuticals, Bristol Myers Squibb (BMS), Constellation Pharma, Exelixis, Inc., Flatiron, Janssen Pharma, Merck Sharp \& Dohme, Michael J Hennessey Assoc, Myovant Sciences, Inc., Physician Education Resource, LLC, Pfizer, Propella Therapeutics, Revhealth, LLC, Sanofi, and Vizuri Health Sciences; has been on advisory boards for Astellas, AstraZeneca, Capio Biosciences, and Modra 
Pharma; has received institutional research grants from Astellas, BMS, Calithera, Exelixis, Inc., Janssen Pharma, Novartis, Pfizer, and Sanofi; has been a steering committee member for AstraZeneca, NCl Genitourinary, and Nektar Therapeutics; has been an independent contractor with Axess Oncology; has been a speaker for Bayer Pharmaceuticals, Exelixis, Inc., and Sanofi; has served on a speakers' bureau for Exelixis, Inc.; has received honoraria from EMD Serono, Ipsen, Michael J Hennessey Assoc, UroGPO, and UroToday; and has received travel accommodation fees from Bayer Pharmaceuticals, Exelixis, Inc., Sanofi, and UroToday.

\section{Author details}

${ }^{1}$ Barts Cancer Institute, Queen Mary University of London, London, UK. ${ }^{2}$ Dana-Farber Cancer Center, Boston, MA, USA. ${ }^{3}$ Memorial Sloan Kettering Cancer Center, New York, NY, USA. ${ }^{4}$ University of Texas MD Anderson Cancer Center, Houston, TX, USA. ${ }^{5}$ City of Hope National Medical Center, Duarte, CA, USA. ${ }^{6}$ Brigham and Women's Hospital, Boston, MA, USA. 'Exelixis, Inc, Alameda, CA, USA. ${ }^{8}$ Gustave-Roussy, Villejuif, France. ${ }^{9}$ Duke Cancer Institute, Durham, NC, USA.

\section{Received: 30 March 2021 Accepted: 16 July 2021 \\ Published online: 07 August 2021}

\section{References}

1. Choueiri TK, Motzer RJ. Systemic therapy for metastatic renal-cell carcinoma. N Engl J Med. 2017;376(4):354-66. https://doi.org/10.1056/NEJMra1601333.

2. Loo V, Salgia M, Bergerot P, Philip EJ, Pal SK. First-line systemic therapy for metastatic clear-cell renal cell carcinoma: critical appraisal of emerging options. Target Oncol. 2019;14(6):639-45. https://doi.org/10.1007/s11523-01 9-00676-y.

3. Choueiri TK, Escudier B, Powles T, Mainwaring PN, Rini Bl, Donskov F, et al. Cabozantinib versus everolimus in advanced renal-cell carcinoma. N Engl J Med. 2015;373(19):1814-23. https://doi.org/10.1056/NEJMoa1510016.

4. Choueiri TK, Escudier B, Powles T, Tannir NM, Mainwaring PN, Rini Bl, et al. Cabozantinib versus everolimus in advanced renal cell carcinoma (METEOR): final results from a randomised, open-label, phase 3 trial. Lancet Oncol. 2016;17(7):917-27. https://doi.org/10.1016/S1470-2045(16)30107-3.

5. Choueiri TK, Hessel C, Halabi S, Sanford B, Michaelson MD, Hahn O, et al. Cabozantinib versus sunitinib as initial therapy for metastatic renal cell carcinoma of intermediate or poor risk (Alliance A031203 CABOSUN randomised trial): progression-free survival by independent review and overall survival update. Eur J Cancer. 2018;94:115-25. https://doi.org/10.101 6/j.ejca.2018.02.012.

6. Yakes FM, Chen J, Tan J, Yamaguchi K, Shi Y, Yu P, et al. Cabozantinib (XL184), a novel MET and VEGFR2 inhibitor, simultaneously suppresses metastasis, angiogenesis, and tumor growth. Mol Cancer Ther. 2011;10(12): 2298-308. https://doi.org/10.1158/1535-7163.MCT-11-0264.

7. Nickerson ML, Jaeger E, Shi Y, Durocher JA, Mahurkar S, Zaridze D, et al. Improved identification of von Hippel-Lindau gene alterations in clear cell renal tumors. Clin Cancer Res. 2008;14(15):4726-34. https://doi.org/10.1158/1 078-0432.CCR-07-4921.

8. Shen C, Kaelin WG Jr. The VHL/HIF axis in clear cell renal carcinoma. Semin Cancer Biol. 2013;23(1):18-25. https://doi.org/10.1016/j.semca ncer.2012.06.001.

9. Rankin EB, Fuh KC, Castellini L, Viswanathan K, Finger EC, Diep AN, et al. Direct regulation of GAS6/AXL signaling by HIF promotes renal metastasis through SRC and MET. Proc Natl Acad Sci U S A. 2014;111(37):13373-8. https://doi.org/10.1073/pnas.1404848111.

10. Pennacchietti S, Michieli P, Galluzzo M, Mazzone M, Giordano S, Comoglio PM. Hypoxia promotes invasive growth by transcriptional activation of the met protooncogene. Cancer Cell. 2003;3(4):347-61. https://doi.org/10.1016/ S1535-6108(03)00085-0.

11. Gibney GT, Aziz SA, Camp RL, Conrad P, Schwartz BE, Chen CR, et al. C-Met is a prognostic marker and potential therapeutic target in clear cell renal cell carcinoma. Ann Oncol. 2013;24(2):343-9. https://doi.org/10.1093/annonc/mds463.

12. Gustafsson A, Martuszewska D, Johansson M, Ekman C, Hafizi S, Ljungberg B, et al. Differential expression of Axl and Gas6 in renal cell carcinoma reflecting tumor advancement and survival. Clin Cancer Res. 2009;15(14): 4742-9. https://doi.org/10.1158/1078-0432.CCR-08-2514

13. Zhou L, Liu XD, Sun M, Zhang X, German P, Bai S, et al. Targeting MET and $\mathrm{AXL}$ overcomes resistance to sunitinib therapy in renal cell carcinoma. Oncogene. 2016;35(21):2687-97. https://doi.org/10.1038/onc.2015.343.
14. Heng DY, Xie W, Regan MM, Warren MA, Golshayan AR, Sahi C, et al. Prognostic factors for overall survival in patients with metastatic renal cell carcinoma treated with vascular endothelial growth factor-targeted agents: results from a large, multicenter study. J Clin Oncol. 2009;27(34):5794-9. https://doi.org/10.1200/JCO.2008.21.4809.

15. Bui MH, Seligson D, Han KR, Pantuck AJ, Dorey FJ, Huang Y, et al. Carbonic anhydrase IX is an independent predictor of survival in advanced renal clear cell carcinoma: implications for prognosis and therapy. Clin Cancer Res. 2003;9(2):802-11.

16. Tran HT, Liu Y, Zurita AJ, Lin Y, Baker-Neblett KL, Martin AM, et al. Prognostic or predictive plasma cytokines and angiogenic factors for patients treated with pazopanib for metastatic renal-cell cancer: a retrospective analysis of phase 2 and phase 3 trials. Lancet Oncol. 2012;13(8):827-37. https://doi. org/10.1016/S1470-2045(12)70241-3.

17. Pena C, Lathia C, Shan M, Escudier B, Bukowski RM. Biomarkers predicting outcome in patients with advanced renal cell carcinoma: results from sorafenib phase III Treatment Approaches in Renal Cancer Global Evaluation Trial. Clin Cancer Res. 2010;16(19):4853-63. https://doi.org/10.1158/10780432.CCR-09-3343.

18. Escudier B, Eisen T, Stadler WM, Szczylik C, Oudard S, Staehler M, et al. Sorafenib for treatment of renal cell carcinoma: final efficacy and safety results of the phase III Treatment Approaches in Renal Cancer Global Evaluation Trial. J Clin Oncol. 2009;27(20):3312-8. https://doi.org/10.1200/ JCO.2008.19.5511.

19. Voss MH, Chen D, Marker M, Hakimi AA, Lee CH, Hsieh JJ, et al. Circulating biomarkers and outcome from a randomised phase II trial of sunitinib vs everolimus for patients with metastatic renal cell carcinoma. Br J Cancer. 2016;114(6):642-9. https://doi.org/10.1038/bjc.2016.21.

20. Harmon CS, DePrimo SE, Figlin RA, Hudes GR, Hutson TE, Michaelson MD, et al. Circulating proteins as potential biomarkers of sunitinib and interferon-alpha efficacy in treatment-naive patients with metastatic renal cell carcinoma. Cancer Chemother Pharmacol. 2014;73(1):151-61. https://doi org/10.1007/s00280-013-2333-4.

21. Choueiri TK, Powles T, Burotto M, Escudier B, Bourlon MT, Zurawski B, et al. Nivolumab plus cabozantinib versus sunitinib for advanced renal-cell carcinoma. N Engl J Med. 2021;384(9):829-41. https://doi.org/10.1056/ NEJMoa2026982.

22. Tolaney SM, Ziehr DR, Guo H, Ng MR, Barry WT, Higgins MJ, et al. Phase II and biomarker study of cabozantinib in metastatic triple-negative breast cancer patients. Oncologist. 2017;22(1):25-32. https://doi.org/10.1634/ theoncologist.2016-0229.

23. Leibowitz-Amit R, Pintilie M, Khoja L, Azad AA, Berger R, Laird AD, et al. Changes in plasma biomarkers following treatment with cabozantinib in metastatic castration-resistant prostate cancer: A post hoc analysis of an extension cohort of a phase II trial. J Transl Med. 2016;14(1):1-8.

24. Kontovinis LF, Papazisis KT, Touplikioti P, Andreadis C, Mouratidou D, Kortsaris AH. Sunitinib treatment for patients with clear-cell metastatic renal cell carcinoma: clinical outcomes and plasma angiogenesis markers. BMC Cancer. 2009;9(1):82. https://doi.org/10.1186/1471-2407-9-82.

25. Flaifel A, Xie W, Braun DA, Ficial M, Bakouny Z, Nassar AH, et al. PD-L1 expression and clinical outcomes to cabozantinib, everolimus, and sunitinib in patients with metastatic renal cell carcinoma: analysis of the randomized clinical trials METEOR and CABOSUN. Clin Cancer Res. 2019;25(20):6080-8. https://doi.org/10.1158/1078-0432.CCR-19-1135.

26. Marshall SW. Power for tests of interaction: effect of raising the type I error rate. Epidemiol Perspect Innov. 2007:4(1):4. https://doi.org/10.1186/17425573-4-4

27. D'Aniello C, Berretta M, Cavaliere C, Rossetti S, Facchini BA, Lovane G, et al. Biomarkers of prognosis and efficacy of anti-angiogenic therapy in metastatic clear cell renal cancer. Front Oncol. 2019;9:1400. https://doi.org/1 0.3389/fonc.2019.01400.

28. Lee CH, Motzer RJ, Glen H, Michaelson MD, Larkin J, Minoshima Y, et al. Correlative serum biomarker analyses in the phase 2 trial of lenvatinib-pluseverolimus in patients with metastatic renal cell carcinoma. Br J Cancer. 2020;124:237-46.

\section{Publisher's Note}

Springer Nature remains neutral with regard to jurisdictional claims in published maps and institutional affiliations. 